Part 1.

History of Early Polar Motion Research 


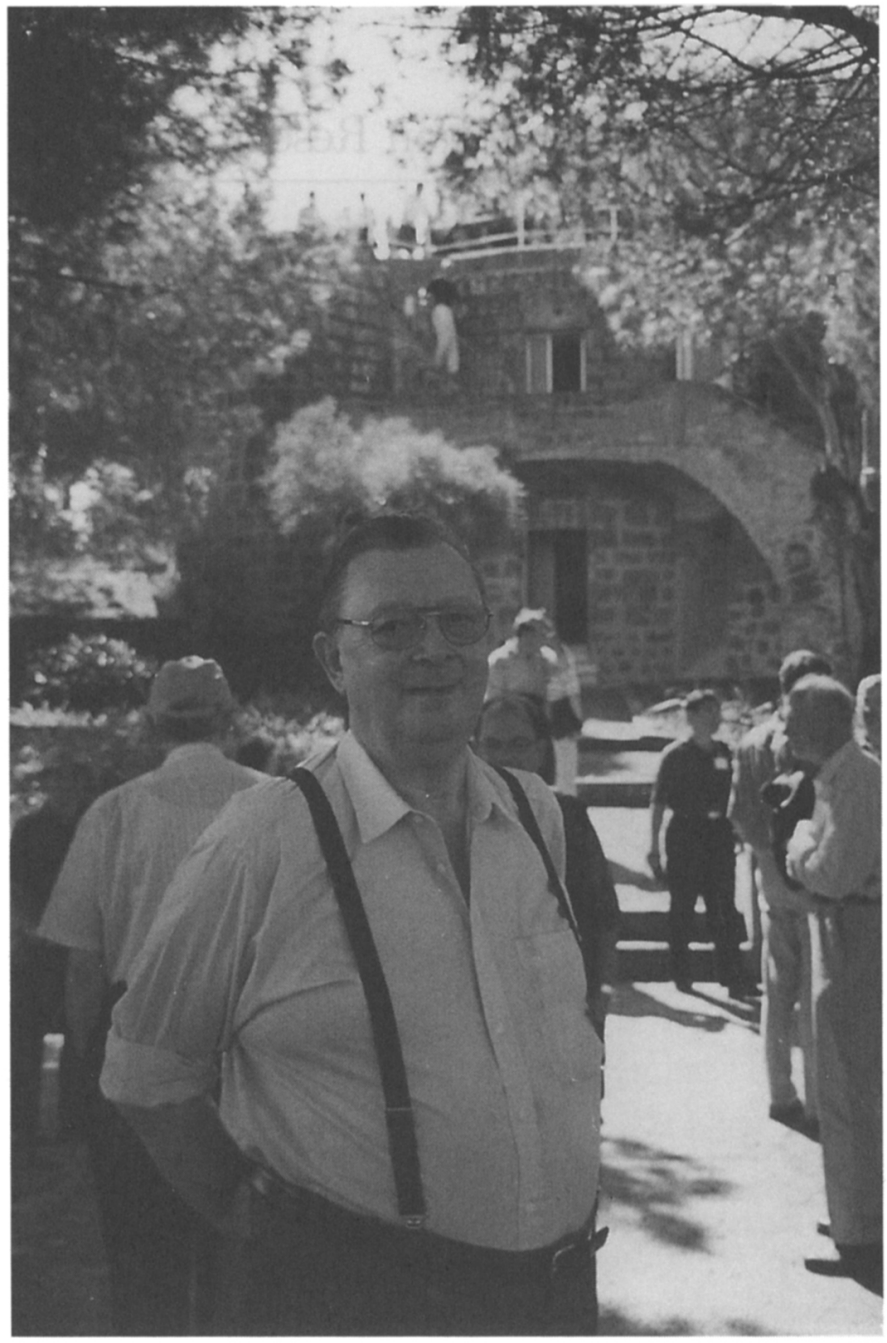

Paul Melchior 


\title{
On Leonhard Euler's Contribution to the Theory of Precession and Nutation
}

\author{
Victor K. Abalakin \\ Pulkovo Observatory, Saint-Petersburg, Russia
}

\begin{abstract}
Among the immense heritage of Leonhard Euler's astronomical, mechanical, and mathematical works there has been particularly strong interest in those that deal with principles describing the Earth's perturbed rotation around its axis. The outstanding meaning of the works is obvious even today, since they give a deep insight into the essence of the phenomena of precessional and nutational displacements of the fundamental coordinate planes and axes constituting astronomical frames of reference.
\end{abstract}

\section{Euler's 1751 Memoir}

In his famous paper entitled "Recherches sur la précession des équinoxes et sur la nutation de l'axe de la. Terre", Euler gives in a very systematic and clear manner his considerations concerning the entire problem of precession and nutation. He begins with Lemma 1 stating that if the Earth is supposed to have a spherical shape of radius $a$ and to be composed of homogeneous matter having the mass $M$, then its moment of inertia with respect to any axis passing through its center will be $2 / 5 M a^{2}$. (Here we have adopted the usual conventional form for $a^{2}$ instead of writing $a a$ as it has been done in times of Euler).

Lemma 1 is followed by the Corollary saying that in spite of the fact that the Earth's shape actually differs slightly from the spherical one, its moment of inertia may nevertheless still be expressed as $2 / 5 M a^{2}$, this expression not being noticeably changed if one takes for $a$ the Earth's semi-axis or the radius of its equator. Then, in the Remark Euler reminds us of the definition of the body's moment of inertia with respect to the axis of the body's rotation, as the sum of products of masses of the body's particles times the squares of their distances from the axis of rotation. Analogously, the expression for the moment of inertia with respect to the axis of rotation has been given as $2 / 5 M\left(a^{5}+\nu \alpha^{5}\right) /\left(a^{3}+\nu \alpha^{3}\right)$ for the case where the spherical Earth of radius $a$ has a spherical core of the radius $\alpha$, their densities being in ratio of $1: 1+\nu$. In Corollary 1 Euler mentions two cases where the core is more or less dense than the Earth's crust according to the sign of the parameter $\nu$, i.e. $>0$ for the first case, and $<0$ for the second one. For the "empty" core space one has $\nu=-1$. Corollary 2 states that neither a slight deviation of the Earth's shape from a sphere, nor that of the core, would affect in a noticeable way the expression of the moment of inertia, and, hence, the precession of equinoxes. 
The Hypothesis contains considerations on conservation of the rate of rotation, and of the spatial direction of the rotation axis, if no external forces are acting on the Earth that could cause any changes in the velocity of its rotation or in the position of its rotation axis. Euler says in his Remark that it is not sufficient for the free rotation of a body that the rotation axis pass through its gravity center. It is also necessary that all centrifugal forces be in equilibrium; otherwise, if the centrifugal forces don't cancel each other, the rotation axis will permanently change its position due to the centrifugal forces, being dependent solely on the Earth's matter distribution. So also will the terrestrial poles even in the absence of any external forces. Further on he is going to show that there are no other changes in the terrestrial pole's positions than those which are caused by forces due to the Sun and the Moon.

In the following Corollary Euler says that any force passing through the Earth's gravitational center changes nothing, either in Earth rotation or in the position of its rotation axis, and it is only in terms of forces acting on the Earth and not passing through its center of gravity that one can explain the changes in the pole's position.

After these introductory paragraphs Euler considers a series of so-called Problems with their Solutions, and accompanying Corollaries and Remarks.

Problem 1 deals with finding the total force acting on the spheroidal and homogeneous Earth and resulting from the attractions of its particles by the fixed mass point $O$, the attractions being proportional to any power $n$ of the distance $z$ (Figure 1). Using his Théorie des forces Euler represents the total force exerted on the Earth as a sum of forces acting along $C O$ and $T O$, with a note that the two forces might be represented as an unique force passing through the mass point $O$ and through some point on the Earth rotation axis lying somewhat below the center $C$. Here we find Euler's statement concerned with the displacement of the point by the force applied from the center of gravity of the attracted body, a matter discussed later by Lamb (1929), and by Yurkina and Bondareva (1993).

In Corollary 1 Euler states that the force $C O$ passing through the Earth's center doesn't contribute anything to Earth rotation or to the changes of its pole positions, so nothing could occur under the action of this force when taken alone. In his Corollary 2 Euler points out that the only force that would be able to produce any changes in Earth rotation and in the position of its poles by its moment is the force directed along TO. And Corollary 3 deals with the expression of this moment as TO.CT. One could, however, imagine an equivalent force $A G$ applied perpendicularly to the rotation axis at $A$ which tends to pull away the point $A$ from the point $O$, the force $A G$ being situated in the plane $A B O$. The moment of this force is represented as $A G . A C$, and will produce the same effect in Earth rotation as the attractive force exerted by $O$. By introducing the apparent angular distance $\phi=A C O$ of the point $O$ from the terrestrial pole $A$, Euler gives a new expression for the moment $A G . A C$ and emphasizes that the moment turns out to be zero when $e=a$, i.e. for a spherical Earth; the moment will be positive or negative for $e>a$ or for $e<a$, correspondingly. The moment will be negative when $e>a$ if the angle $\phi>90^{\circ}$; it will cancel itself for $\phi=90^{\circ}$, and reach the maximum value for $\phi=45^{\circ}$ or $135^{\circ}$. The last results are contained in the Corollaries \& through 7. 


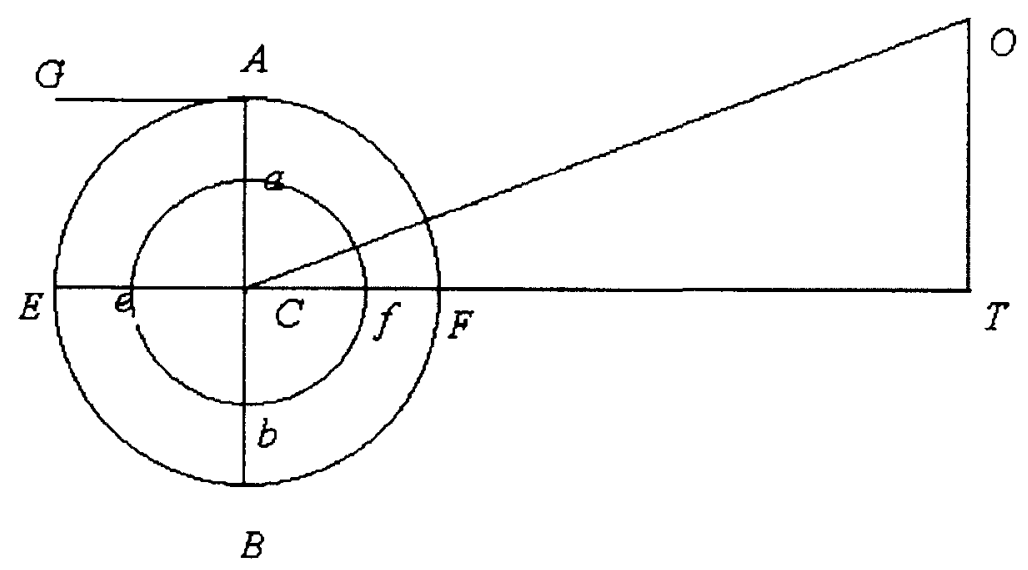

Figure 1. Forces acting on the Earth.

Problem 2 deals with determining the moment of a force exerted on the Earth containing a spheroidal core under attraction of the center of forces $O$, the attractions being proportional to any power of distances. Euler finds the expression for the moment of the force $A G . A C$, and considers it for the case of the Sun putting $n=2$ as well as for the Moon, for which the attractive force had been adopted in the form $k^{2} / h^{2}-\delta, \delta$ being an empirically introduced constant.

Problem 3 is dedicated to determining the instantaneous change in the rotation axis position due to action of the moment of force $A C . A G=S$ considered above. Euler points out that the rate of rotation $d s / d t$ will be unchanged, whereas the rotation will occur around a new axis directed towards the point $a$ that is situated in the plane of the meridian located $90^{\circ}$ away from the meridian containing the force $A G$, and he gives the expression for this change defined by the angle $A C a$ formed in the time $d t$ :

$$
A C a=\left(S d t^{2} / 2 d s\right) /\left((2 / 5) M a^{2}\right)=5 S d t^{2} / 4 M a^{2} d s .
$$

In the accompanying Corollaries 1 through 4 there are observations concerning the changes in position of the Earth's poles in the sky as well as on the surface of the Earth itself, effects of the attractive force center located in the meridian $A F$ opposite to $A E$, and effects of the Earth's core already considered above. In the Remark Euler gives the transformed expression for the instantaneous change of the Earth's pole (the angle $A C a$ ) going from the time $d t$ to the corresponding mean angular motion of the Sun $d v$ and the mean diurnal rotation of the Earth $d s$ and using more known quantities like the mean distance of the Sun from the Earth $b$ and its attraction on the Earth $=c^{2} / b^{2}$, so that $d t^{2}=2 b^{3} / c^{2} d v^{2}$ and $d s=36625 / 81 d v$.

In Problem 4 the elementary change of the Earth's pole in the sky due to the forces exerted separately by the Sun and the Moon should be found. Putting 
for brevity's sake

$$
N=\left(a^{3}\left(e^{2}-a^{2}\right)+v \alpha^{3}\left(\varepsilon^{2}-\alpha^{2}\right) /\left(a^{5}+v \alpha^{5}\right)\right),
$$

and supposing that the North pole of the Earth actually corresponds to the point $A$ in the sky, Euler gets for the change of the pole position

$$
A C a=(3 / 73250 / 81) N d v \sin \phi \cos \phi,
$$

with $h=b, k=c, n=2$ due to the Sun situated in the point $O$.

For the Moon, now taken as the force center, the distance from the Earth is $h$ and the exerting force is $k^{2} / h^{2}-\delta$ so that the value of $(n+1) k^{n} / h^{n+1}$ becomes $3 k^{2} / h^{3}-\delta / h$; therefore, for the change of the pole position one gets

$$
A C a=(3 m / 73250 / 81) N d v \sin \phi \cos \phi,
$$

accounting for the ratio of the tide-generating force of the Moon to that of the Sun $\left(3 c^{2} / b^{3}\right)$ as $m$ to 1 . The following Corollaries 1 and 2 deal with potentialities to get a judgment concerning the Earth's core once the value of $\lambda=(3 / 73250 / 81) N$ is found by use of the observed changes of the pole position. In the Remark Euler points out that if the Earth had no core one could determine $N$ by the use of the meridian arc measurements; since these measurements made in the North (in Lapland), in France, and in Peru have given that

$$
a: e=200: 201
$$

one has

$$
N=(201 / 200)^{2}-1 \simeq 1 / 100,
$$

and

$$
\lambda=3 /(100.73250 / 81)=1 / 24421 .
$$

In Problem 5 the changes in the Earth's pole position are found for the force exerted by the moving Sun. The solution is found for the coordinates $x$ and $y$ of the Earth's pole $P$ with respect to the pole of ecliptic $\Pi$ (Figure 2). After several transforms and reductions given in the Corollaries 1 through 4, Euler provides the expressions for $x$ and $y$ in Corollary 5 assuming the Earth to be homogeneous $(\lambda=1 / 24421$ as shown above)

$$
\begin{gathered}
x=C-24 \frac{1}{3} A^{\prime \prime}+1 \frac{8}{9}^{\prime \prime} \sin 2 p, \\
y=\theta+\frac{5}{6}^{\prime \prime} \cos 2 p,
\end{gathered}
$$

the annual precession of the pole and of the equinoxes as well being equal to 24 $1 / 3$ seconds, $A$ being expressed in years.

Problem 6 deals with finding the changes in the Earth's pole position due to the attraction of the Moon moving uniformly in its geocentric orbit (Figure 3), whereas Problem 7 is dedicated to determination of the Earth's pole position changes (variations) due to the joint action of the Sun and Moon attractive forces. Euler puts $\theta$ for the mean angular distance of the Earth's North pole 


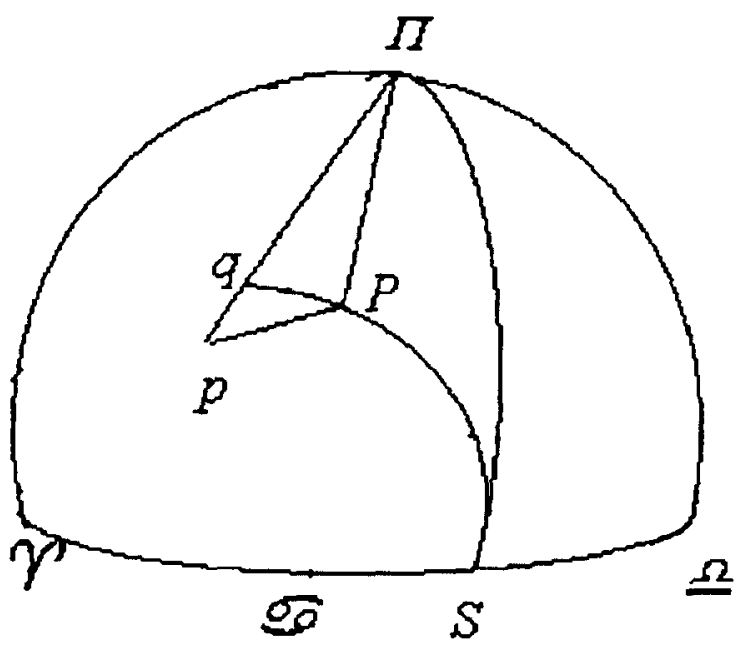

Figure 2. Changes in the Earth's pole position.

from the pole of the ecliptic and assumes the mean longitude of the Earth's pole reckoned from a fixed star, say, $1^{*} \Upsilon$ (it means: from the first star of Aries), to be $=\zeta$. Now one should find the true longitude $x$ and the true latitude $90^{\circ}-y$ which define the position of the Earth's pole. In accordance with the above assumptions one gets

$$
\begin{gathered}
x=\zeta-0.45862 \lambda v+47298^{\prime \prime} \sin 2 p-0.45302 \lambda m v+296527^{\prime \prime} \lambda m \sin u, \text { and } \\
y=\theta+20541^{\prime \prime} \lambda \cos 2 p+158720^{\prime \prime} \lambda m \cos u,
\end{gathered}
$$

so that the mean motion of the pole is given as

$$
x=\zeta-0.45862 \lambda v-0.45302 \lambda m v, \text { and } y=\theta .
$$

One sees that while the mean distance of the Earth's pole from the ecliptic pole remains unchanged, the Earth's pole has retrograde motion in longitude with respect to the stars, making in one year

$$
1296000^{\prime \prime} \lambda(0.45862+0.45302 m)=594371^{\prime \prime} \lambda+587114^{\prime \prime} \lambda m \text {. }
$$

Making use of James Bradley's observations Euler finds approximately

$$
594371^{\prime \prime} \lambda+587114^{\prime \prime} \lambda m=50.5 \text {, and } 158720^{\prime \prime} \lambda m=9^{\prime \prime},
$$

from which it follows $m=2$; for this ratio of the tide generating forces of the Moon and of the Sun Euler finds the precise annual precession of the Earth's pole 


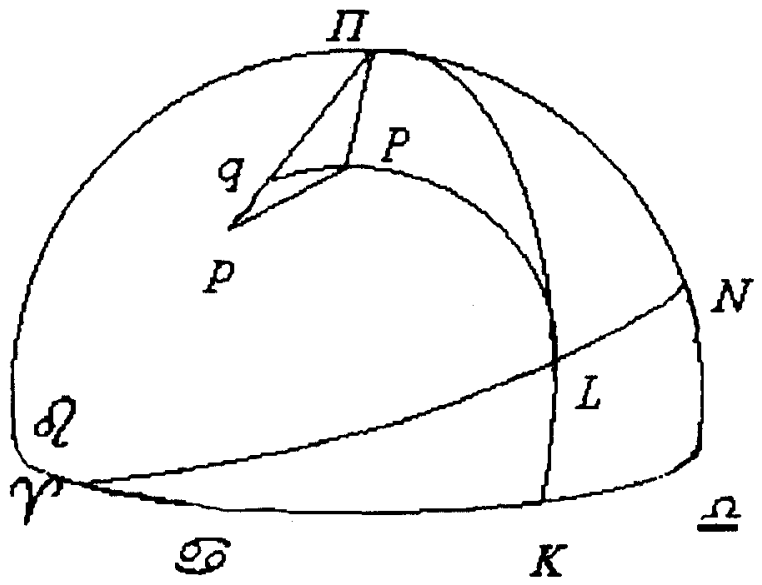

Figure 3. Changes in the Earth's pole position due to the attraction of the Moon.

$=50$ " 3 . This corresponds to the value of $\lambda=1 / 40997$, which is considerably smaller than $\lambda=1 / 24421$ determined for the homogeneous Earth, and speaks in favour of the existence of a central core, its radius $\alpha$ being approximately defined by:

$$
3 r \alpha^{5}=2 a^{5}
$$

e.g., for $1+\nu=10$ one gets $\alpha \approx(3 / 5) a$. Euler says in conclusion of this paragraph that the result ne paroit contraire a aucunes des Observations, qui regardent l'interieur de la terre: il semble qu' un tel noyau est très conforme aux principes de la Physique.

Problem 8 deals with the determination of the longitude and the latitude of the Earth's northern pole for a given moment of time, the longitude being reckoned from a given fixed star. If $\eta$ is the mean longitude of the pole with respect to the first star of Aries for a certain time moment, and $\theta$ is the mean distance of the Earth's pole from that of the ecliptic, being estimated as $23^{\circ} 28^{\prime} 30^{\prime \prime}$, then, for the coordinates $x$ and $y$ of the true position of the pole with $\lambda=1 / 40997$ and $\lambda m=1 / 16399$ one has

$$
\begin{gathered}
x=\eta+18^{\prime \prime} .08 \sin u+1^{\prime \prime} .13 \sin 2 p, \\
y=\theta+9.68 \cos u+0.50 \cos 2 p,
\end{gathered}
$$

where $u$ is the longitude of the ascending node of the Moon's orbit reckoned from the point of the vernal equinox, and $p$ is the longitude of the Sun $[\odot]$ reckoned from the same origin. 
The practical applications may be made on the basis of two tables given in Remark 1, if one wants to find the true longitude of a star, its mean longitude being known. The First Correction to the star's mean longitude at a given time may be found from the first table using the longitude of the ascending node of the Moon's orbit as the argument changing within each Zodiacal sign from $0^{\circ}$ through $30^{\circ}$, being negative for signs 0 through 5 , and positive for signs 6 through 11 ; it means that if the longitude of the ascending node at the time is equal to, say, $285^{\circ}$ the First Correction value is $+17^{\prime \prime} 29^{\prime \prime \prime}$ and should be added to the mean longitude value. The Second Correction is to be taken with the longitude of the Sun as the argument from the second table arranged in the same way as the first one. The accompanying Corollaries 6 to 8 deal with explanations for various practical cases. Remark 2 deals with the two analogous corrections to be added to the mean obliquity of the ecliptic to find its true value at the epoch given.

Finally, Problem 9 deals with determination of the full amount of precession accumulated in the one-year time span proposed. Euler considers the value of the longitude of the ascending node of the lunar orbit corresponding to the middle of the year given, putting it equal to $s$; then, because the annual motion of the line of nodes is $19^{\circ} 20^{\prime}$, the longitude of the ascending node at the beginning of the given year will be $s+9^{\circ} 40^{\prime}$ whereas that at the end of the year will be $s-9^{\circ} 40^{\prime}$. The longitude of the Sun has the same value for the beginning and for the end of the year as well, contributing nothing to the annual precession. Therefore, for the longitude of a star at the beginning of the year one has:

$$
L-18^{\prime \prime} 08 \sin \left(s+9^{\circ} 40^{\prime}\right)-1.13 \sin 2 p,
$$

while for the end of the year one gets:

$$
L+50^{\prime \prime} 3-18^{\prime \prime} 08 \sin \left(s-9^{\circ} 40^{\prime}\right)-1^{\prime \prime} 13 \sin 2 p,
$$

so that, after all the necessary trigonometric reductions are performed, the expression for the total precession will be $50.3+6 . \prime 07 \cos s$. In the accompanying Corollaries Euler makes the statement that the precession of the equinoxes will be the greatest for the years in the middle of which the ascending node of the lunar orbit is located at the beginning of the constellation of Aries $\Upsilon$, amounting to $56^{\prime \prime}, 37^{\prime \prime \prime}$ or $56^{\prime \prime}, 22^{\prime \prime \prime}$, and it will be the smallest for those in the middle of which the ascending node is located at the beginning of the Libra $\Omega$, amounting to $44^{\prime \prime}, 23^{\prime \prime \prime}$ or $44^{\prime \prime}, 14^{\prime \prime \prime}$. If $u$ is the longitude of the ascending node of the lunar orbit for the beginning of a given year, then, because $u=s+9^{\circ} 40^{\prime}$, one has $s=u-9^{\circ} 40^{\prime}$, and the total amount of precession is $50^{\prime \prime} 3+6^{\prime \prime} \cdot 07 \cos \left(u-9^{\circ} 40^{\prime}\right)$. Euler has computed the precession amounts for the values of the ascending node longitude at the beginnings of the years in accordance to their location with respect to the Zodiacal signs $\Upsilon, \gamma, \ldots, \approx$, $*$ and compiled the table to facilitate the precession calculations. In the table given in Corollary 5 the values of the annual precession are compiled for the years 1745 through 1784 , and should serve for the comparison of observations on the mobility of the Earth's axis with the theory developed in the work under consideration to prove the validity of this theory.

In the paper entitled "Avertissement au sujet des recherches sur la precession des equinoxes," Euler (1752) makes a statement concerning the fact that 
he had composed his "Recherches" after having read the treatise of d'Alembert dedicated to the same subject, and he declares that he doesn't pretend to the glory which is due to d'Alembert for being the first to give the excellent solution of this very important problem. Moreover, Euler points out that this fact should not be forgotten by the scientific community, and adds his recognition of d'Alembert's fundamental achievements in studies on curves possessing the point of return of the second kind, or the bird's beak.

\section{Euler's 1765 Memoir}

Euler continues to discuss the problem of the celestial bodies' rotation in his "Remarques générales sur le mouvement diurne des planètes." After the discovery of the real precession of equinoxes and the nutation of the Earth's axis, he notes the diurnal motion of the Earth can be represented in the following way: while the Earth uniformly rotates around this axis the latter is moving itself, being directed towards various points of the sky, and one might compile the appropriate tables that help to compute for any moment of time the longitudes of the Earth's poles as well as their distances from the poles of the ecliptic. This way seemed initially to be the most natural and convenient one in practice, and one could hardly imagine that it will be subject to great difficulties because of the way to understand this motion. Therefore, Euler asks what is the Earth's axis [of rotation]? One would answer that it is some straight line passing through the Earth's center or rather through its center of gravity around which the Earth rotates. Now a difficult question arises on how one could recognize this line. One turns to the sky where there always are two diametrically opposite points which seem to be immovable and around which the sky seems to rotate with all its stars. These points are called the poles, the straight line connecting them and passing through the Earth's center being called the rotation axis. In order to get a clearer idea about the subject, Euler proposes to substitute the Earth with some planet having more rapid motion of its axis of rotation than the Earth, and considers a rotating spherical body (Figure 4) whose pole $P$ moves in some way whereas its center is immovable. Even in the simplest case where there is no axial rotation, the positions of the four selected points $A, B, C, D$, surrounding the pole $P$ will be different depending whether the pole $P$ will move into its new position $Q$ along the arc $P Q$ directly or via the point $R$.

To avoid these difficulties Euler defines for any moment of time the poles' positions as those of the fixed points in the sky and adds the rate of rotation at the moment of time. The rotation of the body, more precisely, the motion of a point $M$ of its surface, is studied with respect to some concentric spherical fixed shell surrounding the body as shown in Figure 5.

After some geometrical constructions and making use of the nice theorem, as he calls it himself (ce beau théoreme), stating that the area of spherical triangles is equal to the excess of the sum of angles of a triangle over $2 \pi$ and that of a quadrangle is equal to the excess of the sum of its angles over $4 \pi$, Euler derives the following differential equations defining the coordinates $x$ and $y$ of the point $M$ :

$$
\begin{gathered}
d x=d p \cos y-d q \sin p \sin y \\
d y=-v d t+d q \cos p-(d p \sin y+d q \sin p \cos y) / \tan x
\end{gathered}
$$




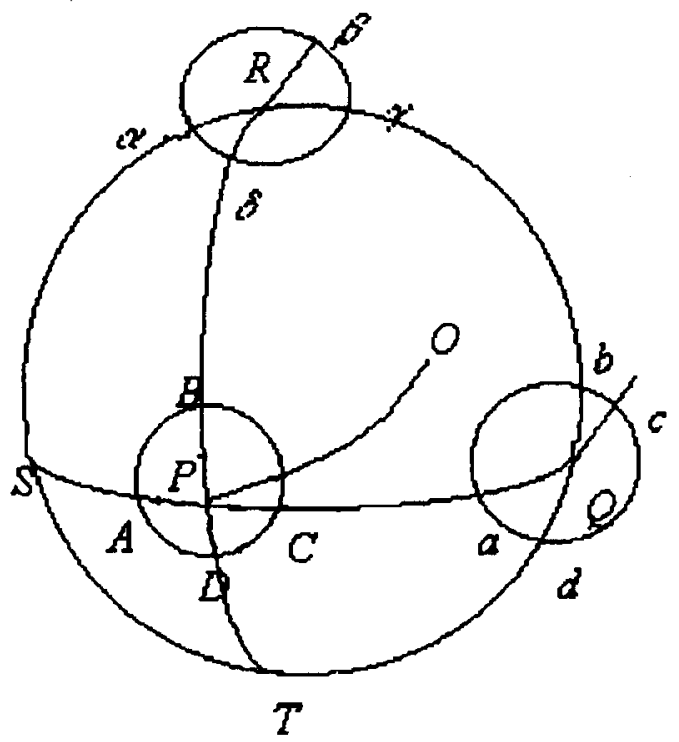

Figure 4. Rotating spherical bodv.

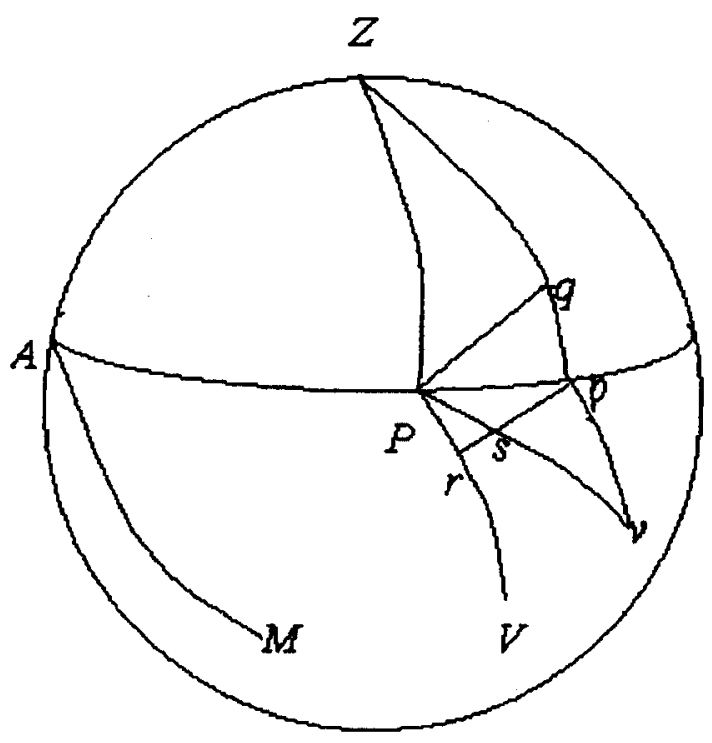

Figure 5. Rotation of a point on the surface of a spherical shell. 


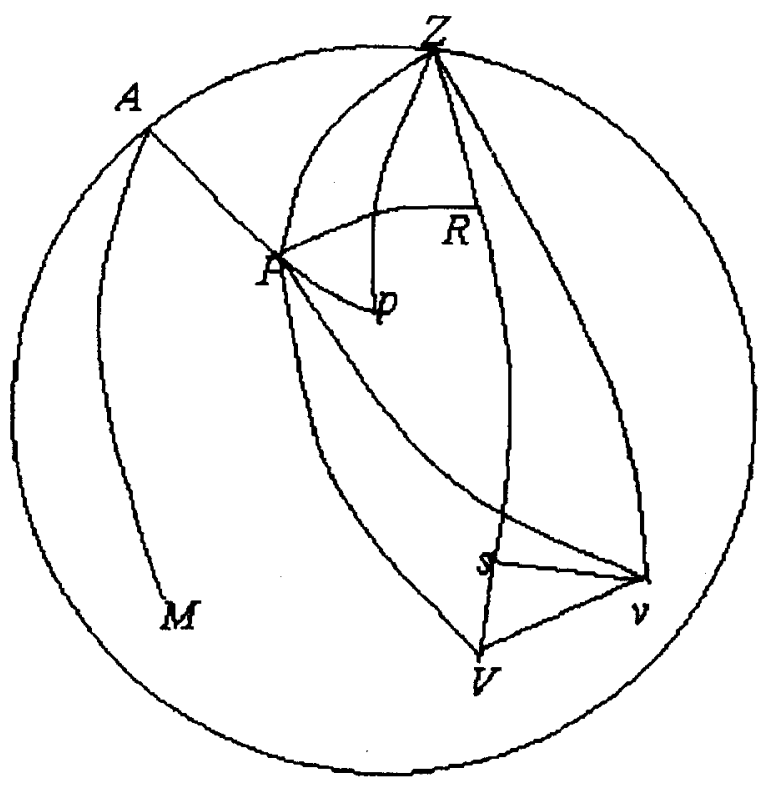

Figure 6. Rotation of a point on the surface of a spherical shell.

These equations may be simplified by introducing the angle $A Z V=u$ and the $\operatorname{arc} Z V=z$ (see Figure 6 ). Some subsequent transforms yield

$$
\begin{gathered}
0=d z+v d t \sin p \sin (u-q), \\
d u \sin z=v d t(\cos p \sin z-\sin p \cos z \cos (u-q)) .
\end{gathered}
$$

Having found $z$ and $u$ one gets

$$
\cos P V=\cos x=\cos p \cos z+\sin p \sin z \cos (u-q),
$$

and

$$
\tan Z P V=\tan y=\sin z \sin (u-q) /(\sin p \cos z-\cos p \sin z \cos (u-q)) .
$$

If there is no rotation, i.e. $v=0$, we see that $d z=0$ and $d u=0$, which means that positions of all the points of the body remain unchanged.

The second case considered by Euler relates to the immovable pole $P$ whatever rotation $v d t$ could be. Thence, $p$ and $q$ should be the constant quantities, and from the first set of equations it follows that $d x=0$ which gives $x=$ const.; 
let $x=f$, and $d y=-v d t$ which means $y=g-\int(v d t)$ whereas the second set would lead to very cumbersome calculations.

Because of complications related to finding the general solution of these equations the two sets will be used depending on the case under consideration. For the Earth one could suppose in the first approximation that the pole $P$ moves uniformly along a small circle around the fixed point $Z$ which represents the pole of the ecliptic, and the rate of the Earth's rotation is permanent all the time.

For this case one has

$$
p=a, \quad d q=m d t, \quad v=n,
$$

and by integration one gets

$$
\begin{gathered}
\left(1+k^{2}\right) \cos x=k^{2} \cos f-\sin f \cos g+(\cos f+k \sin f \cos g) \cos S \\
+\sin f \sin g \sin S\left(1+k^{2}\right) ; \\
\cos y \sin x=k \cos x-k \cos f+\sin f \cos g,
\end{gathered}
$$

where one has put:

$$
k=(n-m \cos a) / m \sin a ; \quad m t \sin a \sqrt{1+k^{2}}=S,
$$

and $f=A M, g=Z A M$ are the starting values of $x$ and $y$. These two formulas define the motion of any point. The point for which the coefficients of $\sin S$ and $\cos S$ are zero may be considered as the proper pole of the planet because its coordinates $x$ and $y$ have constant values, so that it keeps its position with respect to the rotation pole $P$ unchanged and does not participate in the rotation. If one puts $g=180^{\circ}$ one gets for the constant distance $f$ of this point from $P$ :

$$
\tan f=1 / k \text {. }
$$

This distance is equal to only $1^{\prime \prime} / 113$ for the Earth, being almost imperceptible.

In order to find other assumptions about the pole's motion and the rotation for which such an axis could exist Euler puts $x=f$ and $y=g$ and gets conditions of the form

$$
d q \sin p / d p=\text { Const., and } d q \cos p / d p-v d t / d p=\text { Const. }
$$

If these conditions are not fulfilled it would be dubious whether the planet's motion would be reducible to a definite axis. The problem is very complicated and difficult, requiring a simultaneous integration of both differential equations in $d x$ and $d y$.

If one puts

$$
-v+d q \cos p / d t=L, \quad d p / d t=M, \quad d q \sin p / d t=N,
$$

and

$$
\sin x \sin y=r, \quad \sin x \cos y=s,
$$

one gets the equations in the form:

$$
d r=L s d t-N d t \sqrt{1-r^{2}-s^{2}} ; \quad d s=-L r d t+M d t \sqrt{1-r^{2}-s^{2}},
$$


the combination of the two giving

$$
M d r+N d s+L N r d t-L M s d t=0 .
$$

Putting

$$
L d t=d \theta,
$$

the two equations may be integrated if $M=N \tan \theta$ yielding

$$
r \sin \theta+s \cos \theta=\text { Const. }=\sin \gamma
$$

so one finds

$$
\cos x=\cos \gamma \cos \odot, \text { and } y=\tan ^{-1}(M / N)-\tan ^{-1}(\sin \odot / \tan \gamma)
$$

where

$$
\odot=C+\int \sqrt{M^{2}+N^{2}} d t .
$$

If one puts

$$
u=\sqrt{1-r^{2}-s^{2}}
$$

the additional equation can be derived

$$
d u-N r d t+M s d t=0,
$$

which being combined with any of the two equations given above, yields the two more integrable cases. Namely, the second equation together with this additional one may be solved if

$$
\int M d t=\tan ^{-1}(N / L)
$$

and the combination of the third equation with the first one will be integrable if

$$
\int N d t=\tan ^{-1}(L / M) \text {. }
$$

The second case contains that of the Earth but is a much more general one: if $\int M d t=c+p$ and $N / L=\tan \left(\int M d t\right)$, one gets as a solution the expressions for $\cos x$ and $\tan y$. Because the position of the fixed point $Z$ may be arbitrary, the integration could be performed also for the pole's motion considered with respect to any other fixed point. Putting the angle $V P p=z$, one gets $Z P V=$ $y=90^{\circ}+z+p P q$. The equations for $d x$ and $d y$ change into

$$
\begin{gathered}
d r-K s d t=0, \\
d s+K r d t+I u d t=\mathbf{0},
\end{gathered}
$$

the third one being added

$$
d u-I s d t=0, \text { or } I d r-K d u=0,
$$

with

$$
I=\sqrt{M^{2}+N^{2}} \text { and } K=L+(M d N-N d M) /\left(\left(M^{2}+N^{2}\right) d t\right) .
$$


These yield three more integrable cases again: $I=0, K=0$, and $K=m I$.

Euler gives a series of cases of integrability, putting $y=z+T$ where $T$ is any function of time $t$, the integrability conditions being expressed in terms of time-dependent functions $L, M, N$, and $T$. Various new solutions are given in the Problem for more cases described by the equations obtained by the cyclic permutation of variables and functions, and by manifold substitutions of combinations of the functions as well, like putting $L+d P / d t ; M \cos P+\sin P ; N \cos P-$ $M \sin P$ instead of $L ; M$; and $N$.

\section{General Results}

Euler (1766) published more general results of his studies on the rotation of celestial bodies in the paper entitled "Recherches sur le mouvement de rotation des corps célestes". He emphasizes again that the axis of the Earth's figure differs from the rotation axis, never coinciding with the latter because of continuous motion due to nutation and precession.

There are two ways to represent the diurnal motion of the Earth; the first one, adopted in astronomy, consists in choosing a fixed line within the Earth around which the Earth is said to rotate and which is called its axis of rotation, moving itself around the pole of the ecliptic as fixed points in the sky. The second one is the most appropriate in mechanics and consists in marking the points in the sky around which the Earth rotates at every moment in time. This second way of describing the Earth's rotation is uniquely defined by the Earth's motion whereas the first one leads to a manifold of various methods of description. After giving a detailed exposé of his ideas, Euler presents a machine representing this kind of motion (Figure 7). It consists of a circle $P Q R S$ that moves freely around the diametrically opposed pivots $P$ and $R$; at the points $A$ and $D$ on the circle the axis $A D$ of a body $a s d q$ is fixed around which this body can freely rotate whereas the circle is rotating around the pivots $P$ and $R$. To represent the Earth's rotation both rotations should be uniform but each having a different sense of rotation, their angular rotation rates being in the ratio of 1 to $\left(1-b^{2} / a^{2}\right) \cos P A$. The machine may be used to represent the case where all the principal moments of inertia are not equal to each other.

This paper is concluded with profound investigations of the perturbing effects in the precession from the Moon and the Sun.

Acknowledgments. My most cordial thanks are due to Herr Prof. Dr. Erik Grafarend and his colleagues from the Institut für Geodäsie der Universität Stuttgart for sending me a copy of the Technical Report No. 18. 


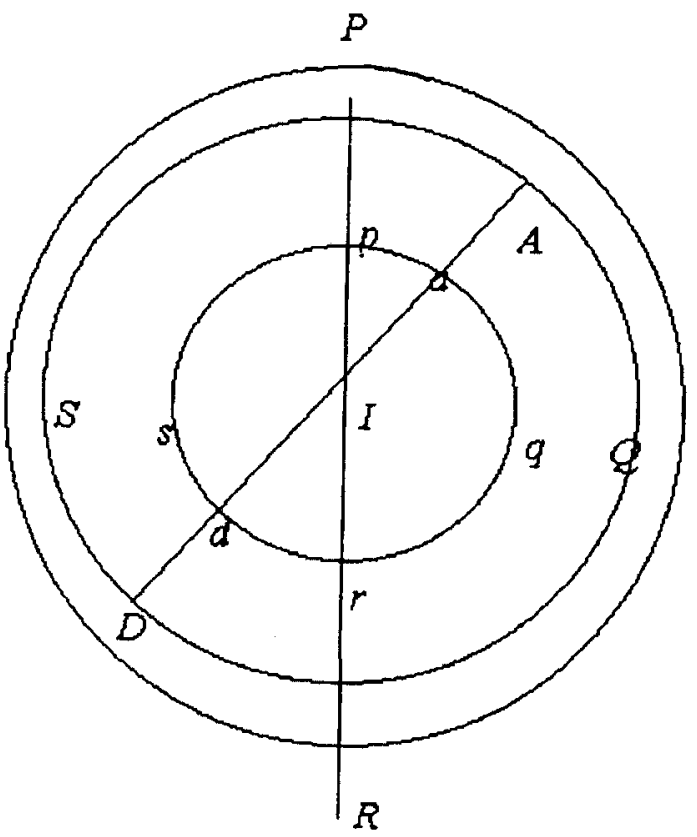

Figure 7. Euler's representation of the Earth's rotation.

\section{References}

Euler, L., Mémoires de l'académie des sciences de Berlin, 1751, p. 289-325.

Euler, L., 1752, p. 412.

Euler, L., 1765, pp. 194-218.

Euler, L., 1766, pp. 265-309.

Lamb, H., Dynamics, Cambridge University Press, 1929.

Yurkina M. I., Bondarewa M. D. Einige Probleme der Erdrotationermittlung. In:

Technical Report No. 18, Institute of Geodesy, University of Stuttgart, 1993. 